\title{
Anatomic study of the position of the mandibular canal and corresponding mandibular third molar on cone-beam computed tomography images
}

\author{
Liqun $\mathrm{Gu}^{1,2} \cdot$ Chao $\mathrm{Zhu}^{2} \cdot \mathrm{Kejia} \mathrm{Chen}^{3} \cdot$ Xianchu Liu ${ }^{3} \cdot$ Zhangui Tang $^{2}$
}

Received: 29 March 2017 / Accepted: 12 September 2017 / Published online: 27 October 2017

(c) The Author(s) 2017. This article is an open access publication

\begin{abstract}
Purpose The positional relationship between the mandibular canal and corresponding third molars is a key anatomic factor of inferior alveolar nerve (IAN) injury. The aim of the present study is to classify the anatomic three-dimensional relationship between the mandibular third molar and the mandibular canal on cone-beam computed tomography (CBCT) images.

Methods This study used CBCT images to classify the positional relationship between the mandibular canal and corresponding third molars. CBCT images of 749 patients (1296 mandibular third molars) were analyzed to draw up a classification.

Results On a total of 1296 third molars, the mandibular canal relative to the roots of the mandibular third molar was on the apical side (88.1\%), followed by the buccal side (7.9\%), the lingual side (3.5\%), and then between the roots $(0.5 \%)$. Ninety-five $(7.1 \%)$ third molars had a close relation with the mandibular canal, while 1201 (92.7\%) third molars had no direct contact. The percentage of the mandibular canal contacts with the mandibular third molar was higher when the mandibular canal was lingually positioned. Conclusions The anatomic structures of the mandibular third molar and the mandibular canal may be helpful to
\end{abstract}

Zhangui Tang

tangzhangui@aliyun.com

1 School of Stomatology, Central South University, Changsha 410008, Hunan, People's Republic of China

2 Department of Oral and Maxillofacial Surgery, Xiangya Stomatology Hospital, Central South University, Changsha 410008, Hunan, People's Republic of China

3 Department of Oral and Maxillofacial Radiology, Xiangya Stomatology Hospital, Central South University, Changsha 410008, Hunan, People's Republic of China make adequate surgical planning to avoid or reduce nerve involvement.

Keywords Mandibular third molar · Inferior alveolar nerve $\cdot$ Cone-beam computed tomography $\cdot$ Anatomic three-dimensional relationship

\section{Introduction}

Extraction of mandibular third molars is the most widely performed oral and maxillofacial surgery. Neurological injury is a rare but serious complication undergoing the operation. The incidence of inferior alveolar nerve (IAN) injury ranges from 0.4 to $6 \%[3,4,30]$. Damage to IAN occurs most frequently when the mandibular third molar has a close relationship with the mandibular canal [29]. Imaging examination is the first step to assess the risk of IAN injury before operation.

The presence of certain radiographic signs on panoramic radiograph indicates a raised risk of IAN involvement. However, panoramic radiograph is observed in two dimensions. There are some overlapping images which make it hard to precisely judge the positional relationship, especially in the buccolingual direction [19, 21, 27].

Recently, cone-beam computed tomography (CBCT) has been widely used in clinical work due to its three-dimensional capability. CBCT not only provides reconstruction images on axial, coronal, and sagittal sections, but also shows the three-dimensional structures of the teeth and surrounding tissues $[1,25]$. The aim of our study is to classify the anatomic three-dimensional relationship between the mandibular third molar and the mandibular canal, which may give a guidance to draw up the surgical plan in case of postoperative complications. 


\section{Materials and methods}

The present anatomic study was approved by the Independent Ethics Committee of the Xiangya Stomatology Hospital of Central South University, People's Republic of China. Informed consent was obtained from all individual participants included in the study. 749 patients who had a CBCT scan and had one or two mandibular third molars with fully formed roots from January 2016 to June 2016 were consecutively included in this study with no restriction of gender. The patients had a mean age of $37.5 \pm 13.6$ years (ranged from 18 to 78 years). 123 patients had one mandibular third molar and 626 patients had two mandibular third molars. Among these molars, 79 third molars were excluded due to (1) residual roots; (2) upward movement due to deficiency of the corresponding maxillary third molar; (3) mesial movement due to deficiency of the adjacent mandibular second molar; (4) fracture and benign or malignant tumor associated with the corresponding mandibular third molar; (5) acute or chronic inflammation in the mandibular third molar zone. Finally, 1296 third molars were enrolled in this study.

The images were acquired using a CBCT scanner (Planmeca, Finland) with the following technical parameters: 90.0-96.0 kV, 10.0-12.0 mA, scan time $<16 \mathrm{~s}$, and FOV of $100 \mathrm{~mm} \times 90 \mathrm{~mm}$. Voxel size was 150 or $400 \mu \mathrm{m}$ and slice thickness of axial images was $0.20 \mathrm{~mm}$. Images were processed using Planmeca Romexis software (Planmeca, Finland) to create axial, coronal, and sagittal reformatted images. Subsequently, the images were analyzed by two clinical radiologists with experience in the field of oral and maxillofacial radiology to classify the three-dimensional relationship of the mandibular third molar and the mandibular canal. If the results were controversial, a senior professor would join the discussion and the final results would be confirmed by the three people.

Position of the mandibular canal relative to the roots of the mandibular third molar is defined as follows (Fig. 1a-n):

Class I: the mandibular canal locates on the apical side (apical position).

Class II: the mandibular canal locates on the buccal side (buccal position).

Class III: the mandibular canal locates on the lingual side (lingual position).

Class IV: the mandibular canal locates between the roots (interradicular position).

Contact relation of the mandibular third molar and the mandibular canal in each class is classified into four conditions (Fig. 1a-n).
Fig. 1 Classification of position and contact relation of the mandibular canal in relation to the mandibular third molar. Position of the mandibular canal in relation to the roots of the mandibular third molar was classified into four classes. Class I: apical position a nocontact; $\mathbf{b}$ contact with a complete white line; $\mathbf{c}$ contact with a defective white line; $\mathbf{d}$ penetration of the mandibular canal. Class II: buccal position e no contact; $\mathbf{f}$ contact with a complete white line; $\mathbf{g}$ contact with a defective white line; $\mathbf{h}$ penetration of the mandibular canal. Class III: lingual position (i) no contact; (j) contact with a complete white line; $\mathbf{k}$ contact with a defective white line; $\mathbf{I}$ penetration of the mandibular canal. Class IV: interradicular position $\mathbf{m}$ contact with a defective white line; n penetration of the mandibular canal. No contact and contact with a complete white line of interradicular position were not found in this study (arrow indicates the mandibular canal)
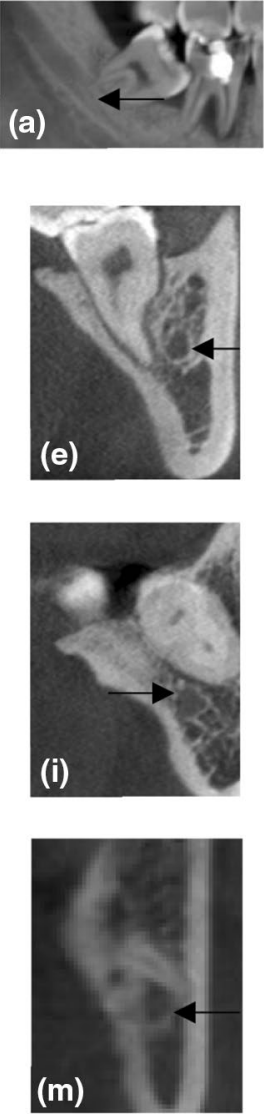
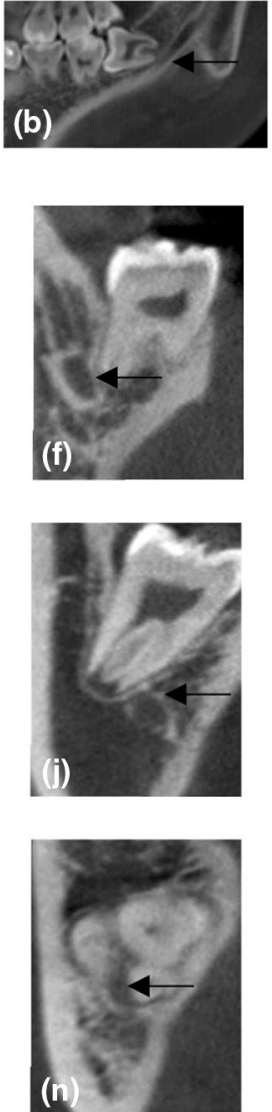
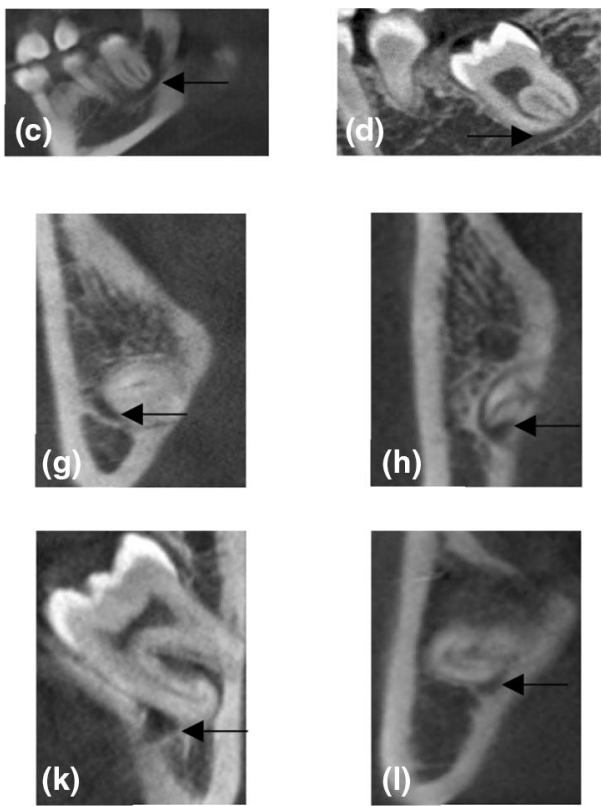
1. The mandibular third molar has no contact with the mandibular canal.

2. The mandibular third molar contacts with the mandibular canal with a complete white line.

3. The mandibular third molar contacts with the mandibular canal with a defective white line.

4. The mandibular third molar penetrates the mandibular canal.

Vertical position of the mandibular third molar and the mandibular canal is categorized into two conditions according to the penetration depth of the roots: the root at the upper half of the mandibular canal; the root at the lower half of the mandibular canal (Fig. 2).

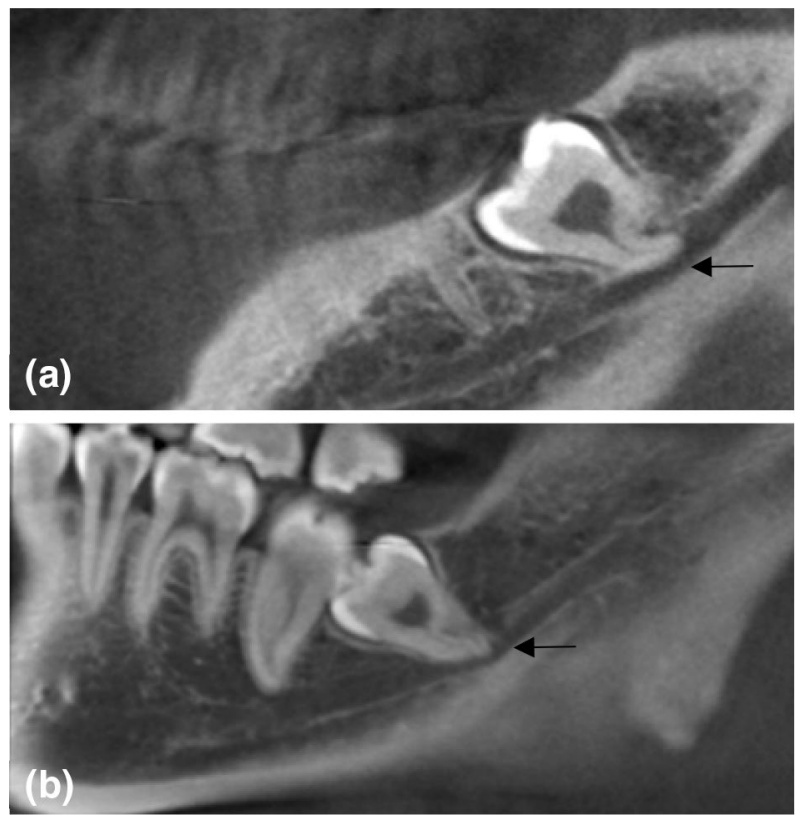

Fig. 2 Vertical position of the mandibular third molar and the mandibular canal according to the penetration depth. Vertical status of the mandibular third molarand the mandibular canal was categorized in twoconditions: a the root at the upper half of themandibular canal; b the root at the lower half ofthe mandibular canal (arrow indicates themandibular canal)

\section{Statistical analysis}

The difference in the percentage of the three-dimensional position of the mandibular canal and corresponding third molars was evaluated by Pearson Chi-square test and Fisher's exact test. $P<0.05$ was regarded as significant. All statistical analysis was performed by SPSS software (Version 20.0.0).

\section{Results}

In the assessment of the position of the mandibular canal in relation to the mandibular third molar on CBCT images, the inter-observer agreement was substantial (kappas of 0.779 for the position of the mandibular canal, and 0.71 for contact relation of the mandibular third molar and the mandibular canal).

The classification was drawn up according to (1) horizontal relationship between the mandibular third molar and the mandibular canal; (2) vertical relationship between the mandibular third molar and the mandibular canal; (3) the integrity of the mandibular canal wall.

Table 1 shows the anatomic position of the mandibular canal relative to the mandibular third molar in the whole study population. On a total of 1296 third molars, the mandibular canal in relation to the roots of the mandibular third molar was on the apical side ( $88.1 \%)$, followed by the buccal side $(7.9 \%)$, the lingual side (3.5\%), and then between the roots $(0.5 \%)$. Table 2 shows that $95(7.3 \%)$ third molars had a direct contact with the mandibular canal, while 1201 (92.7\%) third molars had not. With respect to no contact with the mandibular molar, the mandibular canal tended to be on the apical side. When the mandibular canal contacts with the mandibular third molar, the percentage of the mandibular canal on the lingual side of the mandibular third molar was higher, compared to the "no contact" group $(p<0.05)$. Table 3 shows the different types of contact in Class II and Class III and no significant correlation was found $(p>0.05)$. Table 4 shows the vertical status of the mandibular third molar and the mandibular canal; Thirty-nine third molars were recorded and classified into two groups according to

Table 1 Position of the mandibular canal relative to the mandibular third molar

\begin{tabular}{lrrrr}
\hline & No contact $(n)$ & \begin{tabular}{l} 
Contact $(n)$ \\
\cline { 3 - 5 }
\end{tabular} & & $\begin{array}{l}\text { Contact with a com- } \begin{array}{l}\text { Contact with a } \\
\text { plete white line }\end{array} \\
\text { defective white line } \begin{array}{l}\text { Penetration of the } \\
\text { mandibular canal }\end{array}\end{array}$ \\
\hline Class I: the mandibular canal locates on the apical side & 1110 & 12 & 10 & 10 \\
Class II: the mandibular canal locates on the buccal side & 82 & 5 & 5 & 10 \\
Class III: the mandibular canal locates on the lingual side & 9 & 4 & 5 & 16 \\
Class IV: the mandibular canal locates between the roots & 0 & 0 & 1 \\
\hline
\end{tabular}


Table 2 Contact relation of the mandibular third molar and the mandibular canal in each class

\begin{tabular}{llll}
\hline & No contact $(n, \%)$ & Contact $(n, \%)$ & Total $(n, \%)$ \\
\hline Classification & & & \\
Class I & $1110(85.6 \%)$ & $32(2.5 \%)^{*}$ & $1142(88.1 \%)$ \\
Class II & $82(6.3 \%)$ & $20(1.5 \%)$ & $102(7.9 \%)$ \\
Class III & $9(0.7 \%)$ & $37(2.9 \%)^{* *}$ & $46(3.5 \%)$ \\
Class IV & $0(0 \%)$ & $6(0.5 \%)$ & $6(0.5 \%)$ \\
Total & 1201 & 95 & 1296 \\
\hline
\end{tabular}

*Significant difference in respect of "no contact" group $(p<0.05)$

**Significant difference in respect of "no contact" group $(p<0.05)$

the penetration depth in the mandibular canal. No significant correlation was found $(p>0.05)$.

\section{Discussion}

Removal of the mandibular third molar is one of the most common oral and maxillofacial operations. IAN injury is a serious complication after extraction of the mandibular third molar, affecting the function of the stomatognathic system and the quality of life of patients. The horizontal and vertical positions of the mandibular canal and corresponding third molars is a key anatomic factor of IAN injury [13, 18, 23]. Pre-operational analysis and evaluation are helpful to make a reasonable surgical management to avoid or reduce complications occurrence.

Panoramic radiography is routinely performed in clinical practice before extraction of the mandibular third molar to evaluate the risk of IAN injury [27]. Some radiographic features indicate that there is an increased risk of nerve damage associated with removal of the corresponding mandibular third molar $[5,13,26]$. However, it is not accurate to evaluate the relationship between the mandibular third molar and the mandibular canal in the buccolingual direction $[19,21$, 27].

In this study, 95 (7.3\%) third molars had a close relationship with the mandibular canal. Of these cases, a higher percentage was observed when the mandibular canal was on the lingual side of the mandibular third molar $(p<0.05)$.
Table 4 Vertical status of the mandibular third molar and the mandibular canal

\begin{tabular}{lllr}
\hline & \multicolumn{2}{l}{ Depth of penetration $(n, \%)$} & Total \\
\cline { 2 - 3 } & At the upper half & At the lower half & \\
\hline Classification & & $2(5.4 \%)$ & 10 \\
Class I & $8(21.6 \%)$ & $4(10.8 \%)$ & 10 \\
Class II & $6(16.2 \%)$ & $9(24.3 \%)$ & 16 \\
Class III & $7(18.9 \%)$ & $0(0 \%)$ & 1 \\
Class IV & $1(2.7 \%)$ & 15 & 37 \\
Total & 22 & & \\
\hline
\end{tabular}

The previous study demonstrated that there is an increasing potential of IAN injury when the mandibular canal is situated lingually [9]. In addition, the mandibular third molar had a higher possibility to locate on the lingual side of the jaw [7]. We may hypothesize that lingually positioned mandibular canal is more likely to contact with the mandibular third molar due to insufficient space, as well as interradicular positioned mandibular canal. Although the prevalence of IAN injury is low, regarding a large number of population in China, the absolute number of possible nerve damage is significant. To reduce the risk of IAN injury, several operation techniques have been proposed.

The technique of coronectomy was to separate the crown completely while retaining the roots in the alveolar fossa. The remaining roots lied at least $3 \mathrm{~mm}$ below the crest of alveolar bone and the pulp was left untouched [20]. After surgery, root remnants had the ability to migrate away from the mandibular canal due to new bone formation above the roots, which reduced the possibility of IAN injury undergoing a necessary second operation [6]. The movement of remaining roots reached high rate at 6 months after operation and gradually stopped at 12-24 months postoperatively [14]. Unless the patient became symptomatic, the root remnants were considered as healthy to some extent [24].

Orthodontic extraction referred to orthodontic method to tract mandibular third molars and extraction of the teeth were performed consequently when the roots were far away from the IAN canal $[2,17]$. A high risk of periodontal defect of the adjacent second molar on the distal surface was associated with this method [22]. Time-consuming and costly were also the drawbacks of this method.
Table 3 Different types of contact in Class II and Class III

\begin{tabular}{lllr}
\hline & Class II $(n, \%)$ & Class III $(n, \%)$ & Total \\
\hline Types of contact & & & \\
Contact with a complete white line & $5(8.8 \%)$ & $4(7.0 \%)$ & 9 \\
Contact with a defective white line & $5(8.8 \%)$ & $17(29.8 \%)$ & 22 \\
Penetration of the mandibular canal & $10(17.5 \%)$ & $16(28.1 \%)$ & 26 \\
Total & 20 & 37 & 57 \\
\hline
\end{tabular}


Sagittal split osteotomy was one of the most common operations of mandibular deformity. It was used to remove a lower third molar in close proximity to the mandibular canal $[16,28]$. The advantage was that direct recognition of anatomic structures helped operators to protect IAN, while the disadvantage was that the operation was an extensive and complex procedure.

There were several randomized controlled trials of CBCT versus panoramic radiography to assess the impact on patient outcomes for neural injury. All showed that CBCT did not lead to reduction of nerve injury and other complications, but was superior in predicting the risk of IAN injury $[8,11$, $12,15]$. Consequently, CBCT contributed to more adequate surgical managements [10].

This is a study of Chinese patients. Within the limitation of the present study, the findings may not be generalizable. In addition, prevalence of nerve damage according to the three-dimensional structures is not studied. Therefore, further investigations are needed.

Taken together, the mandibular third molar correlated with higher expectation of nerve involvement when the mandibular canal was lingually situated. Our finding may be helpful to make an adequate surgical plan to avoid or reduce nerve involvement.

Acknowledgements This study was supported by the Department of Oral and Maxillofacial Radiology at Xiangya Stomatology Hospital of Central South University, People's Republic of China.

\section{Compliance with ethical standards}

Conflict of interest The authors declare that they have no conflict of interest.

Open Access This article is distributed under the terms of the Creative Commons Attribution 4.0 International License (http://creativecommons.org/licenses/by/4.0/), which permits unrestricted use, distribution, and reproduction in any medium, provided you give appropriate credit to the original author(s) and the source, provide a link to the Creative Commons license, and indicate if changes were made.

\section{References}

1. Al-Salehi SK, Horner K (2016) Impact of cone beam computed tomography (CBCT) on diagnostic thinking in endodontics of posterior teeth: a before-after study. J Dent 53:57-63. doi:10.1016/j. jdent.2016.07.012

2. Alessandri Bonetti G, Bendandi M, Laino L, Checchi V, Checchi L (2007) Orthodontic extraction: riskless extraction of impacted lower third molars close to the mandibular canal. J Oral Maxillofac Surg 65:2580-2586. doi:10.1016/j.joms.2007.06.686

3. Bui CH, Seldin EB, Dodson TB (2003) Types, frequencies, and risk factors for complications after third molar extraction. J Oral Maxillofac Surg 61:1379-1389. doi:10.1016/j.joms.2003.04.001

4. Cheung LK, Leung YY, Chow LK, Wong MCM, Chan EKK, Fok YH (2010) Incidence of neurosensory deficits and recovery after lower third molar surgery: a prospective clinical study of 4338 cases. Int J Oral Maxillofac Surg 39:320-326. doi:10.1016/j. ijom.2009.11.010

5. de Melo Albert DG, Gomes ACA, do Egito Vasconcelos BC, de Oliveira e Silva ED, Holanda GZ (2006) Comparison of orthopantomographs and conventional tomography images for assessing the relationship between impacted lower third molars and the mandibular canal. J Oral Maxillofac Surg 64:1030-1037. doi:10.1016/j.joms.2006.03.020

6. Dolanmaz D, Yildirim G, Isik K, Kucuk K, Ozturk A (2009) A preferable technique for protecting the inferior alveolar nerve: coronectomy. J Oral Maxillofac Surg 67:1234-1238. doi:10.1016/j.joms.2008.12.031

7. Ge J, Zheng JW, Yang C, Qian WT (2016) Variations in the buccal-lingual alveolar bone thickness of impacted mandibular third molar: our classification and treatment perspectives. Sci Rep 6:16375. doi:10.1038/srep16375

8. Ghaeminia H, Gerlach NL, Hoppenreijs TJM, Kicken M, Dings JP, Borstlap WA, de Haan T, Bergé SJ, Meijer GJ, Maal TJ (2015) Clinical relevance of cone beam computed tomography in mandibular third molar removal: A multicentre, randomised, controlled trial. J Craniomaxillofac Surg 43:2158-2167. doi:10.1016/j.jcms.2015.10.009

9. Ghaeminia H, Meijer GJ, Soehardi A, Borstlap WA, Mulder J, Berge SJ (2009) Position of the impacted third molar in relation to the mandibular canal. Diagnostic accuracy of cone beam computed tomography compared with panoramic radiography. Int J Oral Maxillofac Surg 38:964-971. doi:10.1016/j. ijom.2009.06.007

10. Ghaeminia H, Meijer GJ, Soehardi A, Borstlap WA, Mulder J, Vlijmen OJC, Bergé SJ, Maal TJJ (2011) The use of cone beam CT for the removal of wisdom teeth changes the surgical approach compared with panoramic radiography: a pilot study. Int J Oral Maxillofac Surg 40:834-839. doi:10.1016/j. ijom.2011.02.032

11. Guerrero ME, Botetano R, Beltran J, Horner K, Jacobs R (2014) Can preoperative imaging help to predict postoperative outcome after wisdom tooth removal? A randomized controlled trial using panoramic radiography versus cone-beam CT. Clin Oral Investig 18:335-342. doi:10.1007/s00784-013-0971-x

12. Guerrero ME, Nackaerts O, Beinsberger J, Horner K, Schoenaers J, Jacobs R (2012) Inferior alveolar nerve sensory disturbance after impacted mandibular third molar evaluation using cone beam computed tomography and panoramic radiography: a pilot study. J Oral Maxillofac Surg 70:2264-2270. doi:10.1016/j. joms.2012.04.015

13. Hasegawa T, Ri S, Shigeta T, Akashi M, Imai Y, Kakei Y, Shibuya Y, Komori T (2013) Risk factors associated with inferior alveolar nerve injury after extraction of the mandibular third molar-a comparative study of preoperative images by panoramic radiography and computed tomography. Int J Oral Maxillofac Surg 42:843-851. doi:10.1016/j.ijom.2013.01.023

14. Hatano Y, Kurita K, Kuroiwa Y, Yuasa H, Ariji E (2009) Clinical evaluations of coronectomy (intentional partial odontectomy) for mandibular third molars using dental computed tomography: a case-control study. J Oral Maxillofac Surg 67:1806-1814. doi:10.1016/j.joms.2009.04.018

15. Hutchinson SY (2005) Cone beam computed tomography panoramic images vs. traditional panoramic radiographs. Am J Orthod Dentofacial Orthop 128:550. doi:10.1016/j.ajodo.2005.05.014

16. Jones TA, Garg T, Monaghan A (2004) Removal of a deeply impacted mandibular third molar through a sagittal split ramus osteotomy approach. Br J Oral Maxillofac Surg 42:365-368. doi:10.1016/j.bjoms.2004.02.022

17. Kalantar Motamedi MR, Heidarpour M, Siadat S, Kalantar Motamedi A, Bahreman AA (2015) Orthodontic extraction of 
high-risk impacted mandibular third molars in close proximity to the mandibular canal: a systematic review. J Oral Maxillofac Surg 73:1672-1685. doi:10.1016/j.joms.2015.03.031

18. Kim JW, Cha IH, Kim SJ, Kim MR (2012) Which risk factors are associated with neurosensory deficits of inferior alveolar nerve after mandibular third molar extraction? J Oral Maxillofac Surg 70:2508-2514. doi:10.1016/j.joms.2012.06.004

19. Maegawa H, Sano K, Kitagawa Y, Ogasawara T, Miyauchi K, Sekine J, Inokuchi T (2003) Preoperative assessment of the relationship between the mandibular third molar and the mandibular canal by axial computed tomography with coronal and sagittal reconstruction. Oral Surgery, Oral Medicine, Oral Pathology. Oral Radiol Endod 96:639-646. doi:10.1016/S1079-2104(03)00356-1

20. Martin A, Perinetti G, Costantinides F, Maglione M (2015) Coronectomy as a surgical approach to impacted mandibular third molars: a systematic review. Head Face Med 11:9. doi:10.1186/ s13005-015-0068-7

21. Monaco G, Montevecchi M, Alessandri Bonetti G, Gatto MRA, Checchi L (2004) Reliability of panoramic radiography in evaluating the topographic relationship between the mandibular canal and impacted third molars. J Am Dent Assoc 135:312-318. doi:10.14219/jada.archive.2004.0179

22. Montevecchi M, Incerti Parenti S, Checchi V, Palumbo B, Checchi L, Alessandri Bonetti G (2014) Periodontal healing after 'orthodontic extraction' of mandibular third molars: a retrospective cohort study. Int J Oral Maxillofac Surg 43:1137-1141. doi:10.1016/j.ijom.2014.03.015

23. Nakayama K, Nonoyama M, Takaki Y, Kagawa T, Yuasa K, Izumi K, Ozeki S, Ikebe T (2009) Assessment of the relationship between impacted mandibular third molars and inferior alveolar nerve with dental 3-dimensional computed tomography. J Oral Maxillofac Surg 67:2587-2591. doi:10.1016/j.joms.2009.07.017
24. Pogrel MA, Lee JS, Muff DF (2004) Coronectomy: a technique to protect the inferior alveolar nerve. J Oral Maxillofac Surg 62:1447-1452. doi:10.1016/j.joms.2004.08.003

25. Pohlenz P, Blessmann M, Blake F, Heinrich S, Schmelzle R, Heiland M (2007) Clinical indications and perspectives for intraoperative cone-beam computed tomography in oral and maxillofacial surgery. Oral Surgery, Oral Medicine, Oral Pathology. Oral Radiology Endodontics 103:412-417. doi:10.1016/j. tripleo.2006.05.008

26. Sanmartí-Garcia G, Valmaseda-Castellón E, Gay-Escoda C (2012) Does computed tomography prevent inferior alveolar nerve injuries caused by lower third molar removal? J Oral Maxillofac Surg 70:5-11. doi:10.1016/j.joms.2011.03.030

27. Sedaghatfar M, August MA, Dodson TB (2005) Panoramic radiographic findings as predictors of inferior alveolar nerve exposure following third molar extraction. J Oral Maxillofac Surg 63:3-7. doi:10.1016/j.joms.2004.05.217

28. Sencimen M, Varol A, Gülses A, Altug AH (2009) Extraction of a deeply impacted lower third molar by sagittal split osteotomy. Oral Surgery, Oral Medicine, Oral Pathology. Oral Radiology Endodontology 108:e36-e38. doi:10.1016/j.tripleo.2009.07.003

29. Smith AC, Barry SE, Chiong AY, Hadzakis D, Kha S-L, Mok SC (1997) Inferior alveolar nerve damage following removal of mandibular third molar teeth. A prospective study using panoramic radiography. Aust Dent J 42:149-152. doi:10.1111/j.1834-7819.1997.tb00111.x

30. Valmaseda-Castellón E, Berini-Aytés L, Gay-Escoda C (2001) Inferior alveolar nerve damage after lower third molar surgical extraction: a prospective study of 1117 surgical extractions. Oral Surgery, Oral Medicine, Oral Pathology. Oral Radiol Endodontol 92:377-383. doi:10.1067/moe.2001.118284 